\title{
Duality for constrained robust sum optimization problems
}

\author{
N. Dinh • M.A. Goberna * · D.H. Long • \\ M. Volle \\ To Marco López on the occasion of his 70th anniversary
}

the date of receipt and acceptance should be inserted later

\begin{abstract}
Given an infinite family of extended real-valued functions $f_{i}, i \in I$, and a family $\mathcal{H}$ of nonempty finite subsets of $I$, the $\mathcal{H}$-partial robust sum of $f_{i}$, $i \in I$, is the supremum, for $J \in \mathcal{H}$, of the finite sums $\sum_{j \in J} f_{j}$. These infinite sums arise in a natural way in location problems as well as in functional approximation problems, and include as particular cases the well-known sup function and the so-called robust sum function, corresponding to the set $\mathcal{H}$ of all nonempty finite subsets of $I$, whose unconstrained minimization was analyzed in previous papers of three of the authors [DOI: 10.1007/s11228-019-00515-2 and DOI: 10.1007/s00245019-09596-9]. In this paper, we provide ordinary and stable zero duality gap and strong duality theorems for the minimization of a given $\mathcal{H}$-partial robust sum under constraints, as well as closedness and convex criteria for the formulas on the subdifferential of the sup-function.
\end{abstract}

\footnotetext{
* Corresponding author
}

This research was supported by the National Foundation for Science \& Technology Development (NAFOSTED), Vietnam, Project 101.01-2018.310, and by Ministerio de Ciencia, Innovación y Universidades (MCIU), Agencia Estatal de Investigación (AEI), and European Regional Development Fund (ERDF), Project PGC2018-097960-B-C22.

N. Dinh

International University, Vietnam National University - HCMC,

Linh Trung ward, Thu Duc district, Ho Chi Minh city, Vietnam,

E-mail: ndinh02@gmail.com

M.A. Goberna

Department of Mathematics, University of Alicante, Spain,

E-mail: mgoberna@ua.es

D.H. Long

VNUHCM - University of Science, District 5, Ho Chi Minh city, Vietnam,

and Tien Giang University, Tien Giang town, Vietnam,

E-mail: danghailong@tgu.edu.vn

M. Volle

Avignon University, LMA EA 2151, Avignon, France,

E-mail: michel.volle@univ-avignon.fr 
Keywords Partial robust sums of families of functions - Robust sum optimization problems · stable zero duality gap · stable strong duality theorems $\cdot$ sup-functions

Mathematics Subject Classification: 90C46 49 N15 - 65F20

\section{Introduction}

In previous papers of three of the authors, [10] and [11], we have studied the socalled robust sum $\sum_{i \in I}^{R} f_{i}$ of an infinite family $\left(f_{i}\right)_{i \in I}$ of proper functions from a given locally convex Hausdorff topological vector space (lcHtvs in brief) $X$, called space of decisions, to $\mathbb{R}_{\infty}:=\mathbb{R} \cup\{+\infty\}$, defined as

$$
\sum_{i \in I}^{R} f_{i}(x):=\sup _{J \in \mathcal{F}(I)} \sum_{i \in J} f_{i}(x), \forall x \in X,
$$

where $\mathcal{F}(I)$ denotes the collection of all nonempty finite subsets of $I$. The term "robust sum" is inspired in the fact that, interpreting $\mathcal{F}(I)$ as an uncertainty set for the uncertain optimization problem

$$
\left(\mathrm{P}_{\mathrm{J}}\right) \quad f(x)=\inf _{x \in X} \sum_{i \in J} f_{i}(x),
$$

where the parameter $J$ runs on $\mathcal{F}(I)$, the robust (or pessimistic) counterpart of this parametric problem is the deterministic problem

$$
\text { (RP) } \quad \inf _{x \in X} \sum_{i \in I}^{R} f_{i}(x) \text {. }
$$

Duality and optimality theorems for (RP) and for the result of perturbing its objective function, the robust sum $\sum_{i \in I}^{R} f_{i}$, with a continuous linear functional can be found in [10] and [11], respectively.

We consider in this paper a twofold extension of the theory developed in [11] (i.e., we consider linear perturbations of the objective function): firstly, we replace $\sum_{i \in I}^{R} f_{i}$ with a more general type of functions that could be called partial robust sums and, secondly, we replace unconstrained optimization by constrained one.

Regarding the partial robust sum functions, we associate with each nonempty subfamily $\mathcal{H}$ of $\mathcal{F}(I)$ the $\mathcal{H}$-robust sum of $\left(f_{i}\right)_{i \in I}$, the function $\sum_{i \in I}^{\mathcal{H}} f_{i}$ defined as

$$
\left(\sum_{i \in I}^{\mathcal{H}} f_{i}\right)(x):=\sup _{J \in \mathcal{H}} \sum_{j \in J} f_{j}(x), \forall x \in X,
$$

in other words, we replace the uncertainty set $\mathcal{F}(I)$ by a given infinite subset $\mathcal{H}$ in the definition of robust sum. In contrast with $\sum_{i \in I}^{\mathcal{H}} f_{i}$, which is always well-defined, the limit sum in the sense of nets

$$
\lim _{J \in \mathcal{H}} \sum_{i \in J} f_{i}(x), \forall x \in X,
$$

is not necessarily well-defined, even in the case that $\mathcal{H}$ is a directed by inclusion as $\mathcal{H}=\mathcal{F}(I)$. Note however that, by [10, Lemma 2.5], if $\sup _{i \in I} f_{i}(x) \geq 0$, then

$$
\sum_{i \in I}^{R} f_{i}(x)=\lim _{J \in \mathcal{F}(I)} \sum_{i \in J} f_{i}^{+}(x),
$$


where $f_{i}^{+}(x)=\max \left\{f_{i}(x), 0\right\}$ for all $x \in X$. In particular, $\lim _{J \in \mathcal{H}} \sum_{i \in J} f_{i}(x)$ is welldefined whenever $\mathcal{H}$ is cofinal in the sense that, for any $J \in \mathcal{F}(I)$, there exists $K \in \mathcal{H}$ such that $J \subset K$ (which implies that $\mathcal{H}$ is directed by inclusion, too) and the functions $f_{i}, i \in I$, are non-negative, in which case

$$
\sum_{i \in I}^{\mathcal{H}} f_{i}(x)=\lim _{J \in \mathcal{H}} \sum_{i \in J} f_{i}(x)=\lim _{J \in \mathcal{F}(I)} \sum_{i \in J} f_{i}(x)=\sum_{i \in I}^{R} f_{i}(x), \forall x \in X .
$$

Observe that, when the functions $f_{i}, i \in \bigcup_{J \in \mathcal{H}} J$, are non-negative, given an arbitrary $x \in X$ such that $\sum_{i \in I}^{\mathcal{H}} f_{i}(x)<+\infty$, the set

$$
\left\{i \in \bigcup_{J \in \mathcal{H}} J: f_{i}(x)>0\right\}=\bigcup_{p \in \mathbb{N}}\left\{i \in \bigcup_{J \in \mathcal{H}} J: f_{i}(x) \geq \frac{1}{p}\right\}
$$

is a countable union of finite sets and, so, it is countable. However, since the set in (2) depends on $x$, it is in general impossible to reduce $\sum_{i \in I}^{\mathcal{H}} f_{i}$ to a series function even in the case that $\mathcal{H}$ is cofinal. Observe also that, from [10, Lemma 2.3 ], independently of the sign of the functions $\left(f_{i}\right)_{i \in I}$, one has

$$
\sum_{i \in I}^{R} f_{i}(x) \in \mathbb{R} \Longrightarrow\left\{i \in I: f_{i}(x)>0\right\} \text { is countable. }
$$

If $\mathcal{H}=\mathcal{F}(I), \mathcal{H}$ is obviously cofinal and the corresponding $\mathcal{H}$-robust sum of $\left(f_{i}\right)_{i \in I}$ is nothing else but the robust sum, i.e.,

$$
\sum_{i \in I}^{\mathcal{F}(I)} f_{i}=\sum_{i \in I}^{R} f_{i}
$$

When $I=\mathbb{N}$, the family $\mathcal{H}=\{\{1, \ldots, n\}: n \in \mathbb{N}\}$ is also cofinal and the corresponding $\mathcal{H}$-robust sum of $\left(f_{i}\right)_{i \in \mathbb{N}}$ is the sequential robust sum

$$
\sum_{i \in I}^{\operatorname{seq} R} f_{i}=\sum_{i \in \mathbb{N}}^{\mathcal{H}} f_{i}
$$

which has potential interest in game theory (for instance, in repetitive and sequential games, see, e.g., [30] and [20]).

Given $m \in \mathbb{N}$, the family $\mathcal{H}_{m}$ of all subsets of $I$ of cardinality $m$ is not cofinal and one has

$$
\sum_{i \in I}^{R} f_{i}=\sup _{m \in \mathbb{N}} \sum_{i \in I}^{\mathcal{H}_{m}} f_{i}
$$

In particular, since $\mathcal{H}_{1}$ is the family of all singletons of $I$, we have

$$
\sum_{i \in I}^{\mathcal{H}_{1}} f_{i}=\sup _{i \in I} f_{i}
$$

We now show that $\sum_{i \in I}^{\mathcal{H}_{m}} f_{i}$, for $m=1,2$, can be useful in the location of services with an infinite set of demand points $\left\{x_{i}, i \in I\right\} \subset X$. Let $d: X^{2} \longrightarrow \mathbb{R}_{+}$be a mapping such that, for $x, y \in X, d(x, y)$ represents an estimated distance (or time) between $x$ and $y$. Let $f_{i}(x):=d\left(x, x_{i}\right)$ for all $x \in X$ and $i \in I$. The optimal location at $\bar{x} \in X$ of an emergency service (as a fire station) covering $\left\{x_{i}, i \in I\right\}$ means that 
$\bar{x}$ minimizes the worst distance (or time) between $x$ and the demand points $x_{i}$, $i \in I$, i.e., $\bar{x}$ must be an optimal solution of

$$
\left(\mathrm{RP}_{\mathcal{H}_{1}}\right) \inf _{x \in \mathbb{R}^{2}} \sum_{i \in I}^{\mathcal{H}_{1}} f_{i}(x)
$$

Similarly, if $\bar{x} \in X$ is the optimal location of a unique transfer station (e.g., a transshipments bus station) for a new centralized transport network requiring to go from $x_{i}$ to $x_{j}$ changing at $\bar{x}$, for all $i, j \in I, i \neq j$, then $\bar{x}$ must be an optimal solution of

$$
\left(\mathrm{RP}_{\mathcal{H}_{2}}\right) \inf _{x \in \mathbb{R}^{2}} \sum_{i \in I}^{\mathcal{H}_{2}} f_{i}(x)
$$

The above models would be more realistic by replacing $x \in \mathbb{R}^{2}$ in $\left(\mathrm{RP}_{\mathcal{H}_{1}}\right)$ and $\left(\mathrm{RP}_{\mathcal{H}_{2}}\right)$ by some constraint involving $x$, i.e., by minimizing $\sum_{i \in \mathbb{N}}^{\mathcal{H}_{m}} f_{i}, m=1,2$, subject to suitable constraints.

Regarding the constraints considered in this paper, we assume the existence of a second lcHtvs $Z$ enlarged with a greatest element $+\infty_{Z}$, a nonempty convex cone $S$ in $Z$ which defines a weak ordering on $Z$, and a proper mapping $G$ : $X \longrightarrow Z \cup\left\{+\infty_{Z}\right\}$. Then, we associate with each nonempty subset $\mathcal{H}$ of $\mathcal{F}(I)$ the constrained $\mathcal{H}$-robust sum problem

$$
\begin{aligned}
&\left(\mathrm{RP}_{\mathcal{H}}\right) \inf \sum_{i \in I}^{\mathcal{H}} f_{i}(x) \\
& \text { s.t. } G(x) \in-S .
\end{aligned}
$$

As an illustration of this constrained optimization model, we consider the following functional best approximation problem of a given real-valued function of one variable by polynomials of a limited degree under interpolation and/or side constraints. Let $I$ be a proper interval in $\mathbb{R}$. The problem, inspired in $[13$, Section 4] and [1, Subsection 4.3.3], consists in finding a best $L_{p}$ approximation, on a given subset $T \subset I$ (possibly the whole interval $I$ ), for the $L_{p}$ pseudometric

$$
d(h, g)=\sup _{J \in \mathcal{F}(T)}\left(\sum_{t \in J}|h(t)-g(t)|^{p}\right)^{\frac{1}{p}}, h, g: I \longrightarrow \mathbb{R},
$$

of a given function $h: I \longrightarrow \mathbb{R}$, by means of polynomials of degree less than $n$, say $g(t)=\sum_{k=1}^{n} x_{k} t^{k-1}$ (that can be identified with their vectors of coefficients $\left.x=\left(x_{1}, \ldots, x_{n}\right) \in \mathbb{R}^{n}\right)$ under interpolation conditions of the form $g(t)=h(t)$ for all $t \in U$ and/or side conditions of the form $g(t) \geq h(t)$ for all $t \in V$, with $U, V \subset I$, $U$ having at most $n-1$ elements. In the presence of both types of constraints, $X:=\mathbb{R}^{n}, \mathcal{H}:=\mathcal{F}(T), U \in \mathcal{H}_{1} \cup \ldots \cup \mathcal{H}_{n-1}$, and the approximation problem can be formulated as

$$
\begin{aligned}
\left(\mathrm{RP}_{\mathcal{H}}\right) \quad & \inf \sum_{t \in T}^{\mathcal{F}(T)}\left|h(t)-\sum_{k=1}^{n} x_{k} t^{k-1}\right|^{p} \\
& \text { s.t. } G(x) \in-S,
\end{aligned}
$$

with $Z:=\mathbb{R}^{U} \times \mathbb{R}^{V}$ equipped with the product topology, $G(x)=h(t)-\sum_{k=1}^{n} x_{k} t^{k-1}$ for all $x \in X$ (so that $G: X \longrightarrow Z$ ), and $S:=\left\{0_{U}\right\} \times \mathbb{R}_{+}^{V}$, where $0_{U}$ denotes the null vector in $\mathbb{R}^{U}$.

The closest antecedents of this paper are [10] and [11], which provide duality theorems and results on the structure of the optimal set for the unconstrained 
minimization of the robust sum function $\sum_{i \in I}^{R} f_{i}=\sum_{i \in I}^{\mathcal{F}(I)} f_{i}$. Table 1 , in turn, summarizes the little existing literature, chronologically ordered, on the limit sum function

$$
\sum_{i \in I} f_{i}(x):=\lim _{J \in \mathcal{F}(I)} \sum_{i \in J} f_{i}(x), \forall x \in X .
$$

There, $\mathcal{C}(X)$ and $\Gamma(X)$ represent the sets of continuous functions and lower semicontinuous (lsc in brief) proper convex functions on $X$. The information on $f^{*}$ usually consists in formulas for its epigraph while $\operatorname{argmin}_{X} f$ represents the set of all minima of $f=\sum_{i \in I} f_{i}$ on $X$.

\begin{tabular}{|c|c|c|c|c|c|c|c|}
\hline Paper & Year & $I$ & $f_{i}$ & $X$ & $\partial f$ & $f^{*}$ & $\operatorname{argmin}_{X} f$ \\
\hline$[33]$ & 1998 & $\mathbb{N}$ & $\mathcal{C}(X)$ & Banach & $\checkmark$ & $\checkmark$ & $\checkmark$ \\
\hline$[34]$ & 2004 & any & $\mathcal{C}(X)$ & Banach & $\checkmark$ & $\checkmark$ & $\checkmark$ \\
\hline$[19]$ & 2008 & any & $\Gamma(X)$ & Banach & $\checkmark$ & $\checkmark$ & \\
\hline$[29]$ & 2016 & $\mathbb{N}$ & $\Gamma(X)$ & Banach & $\checkmark$ & $\checkmark$ & $\checkmark$ \\
\hline$[24]$ & 2019 & any & $\overline{\mathbb{R}}^{X}$ & lcHtvs & $\checkmark$ & $\checkmark$ & $\checkmark$ \\
\hline
\end{tabular}

Table 1

The paper is organized as follows. Section 2 introduces the necessary notations and basic concepts together with the perturbation function and the robust dual scheme to be developed along the paper. Section 3 characterizes zero duality gap and strong duality for a fixed linear perturbation $x^{*} \in X^{*}$ of the robust sum function and stable versions of these duality theorems with respect given subsets of $X^{*}$. Section 4 characterizes the subdifferential of the constrained robust sum. Specializing the latter characterization to the unconstrained case with $\mathcal{H}=\mathcal{H}_{1}$ we obtain, in Section 5, Valadier type formulas ([28], [16]) for the subdifferential of the sup function under appropriate closedness criteria (Corollaries 3, 5). It is worth mentioning that generalized versions of Valadier's formula have been obtained by Marco López and his collaborators along the last years (see [5], [7], [6], and references therein).

\section{Preliminaries}

\subsection{Basic notations}

Let $X$ be a lcHtvs with topological dual space $X^{*}$, null vectors $0_{X}$ and $0_{X}^{*}$, respectively, and duality product $\langle\cdot, \cdot\rangle$. We consider $X^{*}$ and $X^{*} \times \mathbb{R}$ equipped with the $w^{*}$-topology and its product by the ordinary topology on $\mathbb{R}$, respectively.

The closure, the convex hull, and the closed convex hull of a subset $A \subset X$ will be denoted by $\bar{A}$, co $A$, and $\overline{\mathrm{co}} A$, respectively. Given $A, B \subset X, A$ is said to be closed (respectively, closed and convex) regarding $B$ if $B \cap \bar{A}=B \cap A$ (respectively, $B \cap \overline{\mathrm{co}} A=B \cap A)$.

The recession cone of a closed convex set $A \subset X$ is

$$
A_{\infty}:=\{v \in X: a+v \in A, \forall a \in A\}=\bigcap_{\alpha>0} \alpha(A-\bar{a})
$$

for any choice of $\bar{a} \in A$. 
Denote $\overline{\mathbb{R}}:=\mathbb{R} \cup\{-\infty,+\infty\}$ and by $\overline{\mathbb{R}}^{X}$ the set of all functions from $X$ to $\overline{\mathbb{R}}$. The definition of $\left(\mathbb{R}_{\infty}\right)^{X}$ is similar. The indicator function of $A \subset X$ is $\delta_{A} \in\left(\mathbb{R}_{\infty}\right)^{X}$ defined by $\delta_{A}(x)=0$ if $x \in A$ and $\delta_{A}(x)=+\infty$ if $x \notin A$. So, $\delta_{A} \in \Gamma(X)$ if and only if $A$ is a nonempty closed and convex subset of $X$.

Given a function $h \in \overline{\mathbb{R}}^{X}$, its domain, its epigraph, its strict epigraph and its Fenchel conjugate are

$$
\begin{gathered}
\operatorname{dom} h:=\{x \in X: h(x)<+\infty\}, \\
\text { epi } h:=\{(x, r) \in X \times \mathbb{R}: h(x) \leq r\}, \\
\operatorname{epi}_{s} h:=\{(x, r) \in X \times \mathbb{R}: h(x)<r\},
\end{gathered}
$$

and $h^{*} \in \overline{\mathbb{R}}^{X^{*}}$ such that

$$
h^{*}\left(x^{*}\right):=\sup \left\{\left\langle x^{*}, x\right\rangle-h(x): x \in X\right\}, \forall x^{*} \in X^{*},
$$

respectively.

The conjugate of a function $\xi \in \overline{\mathbb{R}}^{X^{*}}$ is defined on $X$ by

$$
\xi^{*}(x):=\sup \left\{\left\langle x^{*}, x\right\rangle-\xi\left(x^{*}\right): x^{*} \in X^{*}\right\}, \forall x \in X .
$$

If $\operatorname{dom} \xi^{*} \neq \emptyset$, one has $\xi^{* *}=\left(\xi^{*}\right)^{*}=\overline{\operatorname{co}} \xi$, where $\overline{\operatorname{co}} \xi$ denotes the $w^{*}$-closed convex hull of $\xi$, i.e., epi $(\overline{\mathrm{co}} \xi)=\overline{\mathrm{co}}(\mathrm{epi} \xi)$. If $\xi$ is convex and $\operatorname{dom} \xi^{*} \neq \emptyset$, then $\xi^{* *}$ coincides with the $w^{*}$-closed hull, $\bar{\xi}$, of $\xi$, i.e., epi $\bar{\xi}=\overline{\text { epi } \xi}$.

For $a \in X$, the subdifferential of $h \in \overline{\mathbb{R}}^{X}$ at $a$ is defined as

$$
\partial h(a):= \begin{cases}\left\{x^{*} \in X^{*}: h(x) \geq h(a)+\left\langle x^{*}, x-a\right\rangle, \forall x \in X\right\}, & \text { if } h(a) \in \mathbb{R}, \\ \emptyset, & \text { else. }\end{cases}
$$

One has

$$
x^{*} \in \partial h(a) \Longleftrightarrow\left\langle x^{*}, a\right\rangle-h(a)=h^{*}\left(x^{*}\right) .
$$

For $A \subset X$, the normal cone to $A$ at a point $a \in A$ is defined as

$$
N(A, a):=\partial \delta_{A}(a)=\left\{x^{*} \in X^{*}:\left\langle x^{*}, x-a\right\rangle \leq 0, \quad \forall x \in A\right\} .
$$

The infimal convolution of two functions $g, h \in \overline{\mathbb{R}}^{X}$ is the function $g \square h \in \overline{\mathbb{R}}^{X}$ such that

$$
(g \square h)(x):=\inf \left\{g\left(x_{1}\right)+h\left(x_{2}\right): x_{1}+x_{2}=x\right\} .
$$

Let $Z$ be a second lcHtvs, $S$ be a nonempty convex cone in $Z$, and denote by $\leqq_{S}$ the ordering on $Z$ induced by the cone $S$, i.e.,

$$
z_{1} \leqq_{S} z_{2} \text { if and only if } z_{1}-z_{2} \in-S .
$$

We also enlarge $Z$ by attaching a greatest element $+\infty_{Z}$. Given a map $G: X \rightarrow$ $Z \cup\left\{+\infty_{Z}\right\}$, we define the domain and the epigraph of $G$ to be $\operatorname{dom} G:=\{x \in X$ : $G(x) \in Z\}$, and

$$
\operatorname{epi}_{S} G:=\{(x, z) \in X \times Z: z \in G(x)+S\},
$$

respectively. We assume that $\operatorname{dom} G \neq \emptyset$. 
For $\lambda \in Z^{*}$, we define $\lambda G: X \rightarrow \mathbb{R}_{\infty}$ as

$$
(\lambda G)(x):= \begin{cases}\langle\lambda, G(x)\rangle, & \text { if } x \in \operatorname{dom} G, \\ +\infty, & \text { else. }\end{cases}
$$

Let us consider $F: X \times Z \rightarrow \mathbb{R}_{\infty}$ given by

$$
F(x, z)= \begin{cases}0, & \text { if } G(x)+z \in-S, \\ +\infty, & \text { else, }\end{cases}
$$

i.e., the indicator function of the hypograph of $-G$, that is,

$$
\operatorname{hypo}_{S}(-G):=\{(x, z) \in X \times Z: z \in-G(x)-S\} \text {. }
$$

The conjugate $F^{*}$ of $F$ is the function $F^{*}: X^{*} \times Z^{*} \longrightarrow \mathbb{R}_{\infty}$ such that

$$
F^{*}\left(x^{*}, \lambda\right)= \begin{cases}(\lambda G)^{*}\left(x^{*}\right), & \text { if } \lambda \in S^{+}, \\ +\infty, & \text { else, }\end{cases}
$$

where $S^{+}:=\left\{\lambda \in X^{*}:\langle\lambda, z\rangle \geq 0, \forall z \in S\right\}$ is the dual cone of $S$. Since $F$ is an indicator function, $F^{*}$ is sublinear and epi $F^{*}$ is a convex cone in $X^{*} \times Z^{*} \times \mathbb{R}$. Moreover, denoting by $\operatorname{proj}_{X^{*} \times \mathbb{R}}$ the projection mapping $X^{*} \times Z^{*} \times \mathbb{R} \ni\left(x^{*}, z^{*}, r\right) \longmapsto\left(x^{*}, r\right)$, one has

$$
\operatorname{proj}_{X^{*} \times \mathbb{R}}\left(\text { epi } F^{*}\right)=\bigcup_{\lambda \in S^{+}} \operatorname{epi}(\lambda G)^{*} .
$$

We now state two basic properties related to this set. The first one is consequence of (8) and the fact that epi $F^{*}$ is a convex cone.

Property $1 \bigcup_{\lambda \in S^{+}} \operatorname{epi}(\lambda G)^{*}$ is a convex cone in $X^{*} \times \mathbb{R}$.

Let us define $A:=\{x \in X: G(x) \in-S\}$ and assume that $A \neq \emptyset$.

Property 2 If $\operatorname{epi}_{S} G$ is closed and convex, then

$$
\operatorname{epi} \delta_{A}^{*}=\overline{\bigcup_{\lambda \in S^{+}} \operatorname{epi}(\lambda G)^{*}} .
$$

Proof. Define $\varphi: X^{*} \rightarrow \overline{\mathbb{R}}$ by $\varphi\left(x^{*}\right)=\inf _{\lambda \in Z^{*}} F^{*}\left(x^{*}, \lambda\right)$. Then $\varphi$ is convex (even sublinear) and we have that

$$
\operatorname{epi}_{s} \varphi \subset \bigcup_{\lambda \in S^{+}} \operatorname{epi}(\lambda G)^{*} \subset \operatorname{epi} \varphi .
$$

Consequently,

$$
\operatorname{epi} \bar{\varphi}=\overline{\operatorname{epi} \varphi}=\overline{\bigcup_{\lambda \in S^{+}} \operatorname{epi}(\lambda G)^{*}} .
$$

By definition of $\varphi$ it comes

$$
\varphi^{*}(x)=F^{* *}\left(x, 0_{Z}\right), \forall x \in X .
$$

Since $\operatorname{epi}_{S} G$ is closed and convex, $\operatorname{hypo}_{S}(-G)$ is closed and convex, too, and one has $F=F^{* *}$. By (10) we then have

$$
\varphi^{*}(x)=F\left(x, 0_{Z}\right)=\delta_{A}(x), \forall x \in X,
$$

and $\delta_{A}^{*}=\varphi^{* *}$. Since $\varphi$ is convex and $\operatorname{dom} \varphi^{*}=A \neq \emptyset$, we have $\varphi^{* *}=\bar{\varphi}$, epi $\delta_{A}^{*}=$ epi $\bar{\varphi}$, and, taking (9) into account, we are done. 
Remark 1 Property 2 is often established under the assumption that the mapping $G: X \rightarrow Z \cup\left\{+\infty_{Z}\right\}$ is star $S$-convex lsc [17, Lemma 2.1] in the sense that the next condition holds:

$$
\lambda G \in \Gamma(X), \quad \forall \lambda \in S^{+} .
$$

It is worth observing that, when taking $\lambda=0_{Z^{*}}$ in (11), one must have $\delta_{\operatorname{dom} G} \in$ $\Gamma(X)$ and, in particular, $\operatorname{dom} G$ must be closed, which is a restrictive condition. One can find in [2, Example 3.3] an example of map $G: X \rightarrow Z \cup\{+\infty Z\}$ such that $\operatorname{epi}_{S} G$ is closed and convex while (11) does not hold.

Remark 2 If $\operatorname{epi}_{S} G$ is closed, then $S$ is necessarily closed: picking $a \in \operatorname{dom} G \neq \emptyset$ we have

$$
(\{a\} \times Z) \cap \operatorname{epi}_{S} G=\{a\} \times(G(a)+S),
$$

which is closed. So, $G(a)+S$ and $S$ are closed.

\subsection{Perturbation functions and robust dual scheme}

Let $U$ be an (arbitrary) uncertainty set, and $X, Y_{u}$ be lcHtvs for all $u \in U$. By $0_{u}$ we denote the zero vector in $Y_{u}$. Assume that a perturbation function

$$
F_{u}: X \times Y_{u} \longrightarrow \mathbb{R}_{\infty}
$$

is given for each $u \in U$. We associate with each $x^{*} \in X^{*}$ the robust optimization problem

$$
\left(\mathrm{RP}_{x^{*}}\right) \inf _{x \in X} \sup _{u \in U}\left\{F_{u}\left(x, 0_{u}\right)-\left\langle x^{*}, x\right\rangle\right\},
$$

and its corresponding robust dual problem, defined (following [8] and [9]) as:

$$
\left(\mathrm{RD}_{x^{*}}\right) \sup _{\substack{u \in U \\ y_{u}^{*} \in Y_{u}^{*}}}-F_{u}^{*}\left(x^{*}, y_{u}^{*}\right) .
$$

Denoting by $\operatorname{proj}_{X^{*} \times \mathbb{R}}^{u}$ the projection of $\left(X^{*} \times Y_{u}^{*}\right) \times \mathbb{R}$ on $X^{*} \times \mathbb{R}$, we now consider the qualifying set (introduced in [8] and [9])

$$
\mathcal{Q}:=\bigcup_{u \in U} \operatorname{proj}_{X^{*} \times \mathbb{R}}^{u}\left(\operatorname{epi} F_{u}^{*}\right)
$$

where the novelty, with respect to [18], is that the parameter space $Y_{u}$ in the perturbation function depends on $u \in U$. Let us consider the robust objective function

$$
h(x):=\sup _{u \in U} F_{u}\left(x, 0_{u}\right), \forall x \in X .
$$

Under some suitable closedness and convexity conditions, stable strong robust duality holds for the pair $\left(\mathrm{RP}_{x^{*}}\right)-\left(\mathrm{RD}_{x^{*}}\right)$, i.e.,

$$
\inf \left(\mathrm{RP}_{x^{*}}\right)=\max \left(\mathrm{RD}_{x^{*}}\right) .
$$

In particular, the next characterization of the stable strong duality w.r.t. $\mathcal{V}$ can be derived from [8, Proposition 3.2] (see also [12, Theorem 3.2], [18, Theorem 3.3]). 
Theorem 1 Assume that $F_{u} \in \Gamma\left(X \times Y_{u}\right), u \in U$, $\operatorname{dom} h \neq \emptyset$ and let $\emptyset \neq \mathcal{V} \subset X^{*}$. Next statements are equivalent:

(i) $\inf \left(\mathrm{RP}_{x^{*}}\right)=\max \left(\mathrm{RD}_{x^{*}}\right), \forall x^{*} \in \mathcal{V}$.

(ii) $\mathcal{Q}$ is $w^{*}$-closed and convex regarding $\mathcal{V} \times \mathbb{R}$.

Proof. As $F_{u} \in \Gamma\left(X \times Y_{u}\right)$ for all $u \in U$, it is easy to see that $h(x)=\sup _{u \in U} F_{u}\left(x, 0_{u}\right)=$ $\sup _{u \in U} F_{u}^{* *}\left(x, 0_{u}\right)$ for each $x \in X$, yielding $h^{* *}=\sup _{u \in U} F_{u}^{* *}\left(\cdot, 0_{u}\right)$ (see [12, Proposition 3.1]). The equivalence of $(i)$ and (ii) now follows from [8, Proposition 3.2].

3 Zero duality gap and stable strong duality for constrained robust sum optimization problem

Let $\left(f_{i}\right)_{i \in I}$ be a family of proper functions on $X$, and $\emptyset \neq \mathcal{H} \subset \mathcal{F}(I)$. Let $f:=$ $\sup _{J \in \mathcal{H}} \sum_{i \in J} f_{i}$ and $A=\{x \in X: G(x) \in-S\}$. Given $x^{*} \in X^{*}$, we consider the following constrained robust sum optimization problem:

$$
\left(\mathrm{RP}_{x^{*}}\right) \inf _{x \in A}\left\{f(x)-\left\langle x^{*}, x\right\rangle\right\} .
$$

Throughout this section we assume that the problem $\left(\mathrm{RP}_{x^{*}}\right)$ is feasible, i.e., $A \cap$ $\operatorname{dom} f \neq \emptyset$.

For each $J \in \mathcal{H}$, let $Y_{J}:=X^{J} \times Z$. Consider the perturbation function $F_{J}$ as in (12), i.e., $F_{J}: X \times X^{J} \times Z \rightarrow \mathbb{R}_{\infty}$, defined by

$$
F_{J}\left(x,\left(x_{i}\right)_{i \in J}, z\right)=\sum_{i \in J} f_{i}\left(x+x_{i}\right)+\delta_{-S}(z+G(x)), \forall\left(x,\left(x_{i}\right)_{i \in I}, z\right) \in X \times X^{J} \times Z .
$$

It is clear that, denoting by $0_{J}=\left(0_{X^{J}}, 0_{Z}\right)$ the zero vector in $Y_{J}$, one has

$$
F_{J}\left(x, 0_{J}\right)=F_{J}\left(x, 0_{X_{J}}, 0_{Z}\right)=\sum_{i \in J} f_{i}(x)+\delta_{A}(x), \forall J \in \mathcal{H} .
$$

One then has, for all triple $\left(x^{*},\left(x_{i}^{*}\right)_{i \in J}, \lambda\right) \in X^{*} \times\left(X^{*}\right)^{J} \times Z^{*}$,

$$
F_{J}^{*}\left(x^{*},\left(x_{i}^{*}\right)_{i \in J}, \lambda\right)= \begin{cases}\sum_{i \in J} f_{i}^{*}\left(x_{i}^{*}\right)+(\lambda G)^{*}\left(x^{*}-\sum_{i \in J} x_{i}^{*}\right), & \text { if } \lambda \in S^{+} \\ +\infty, & \text { else }\end{cases}
$$

and the qualifying set defined in (14), $\mathcal{Q}=\bigcup_{J \in \mathcal{H}} \operatorname{proj}_{X^{*} \times \mathbb{R}}^{J}$ epi $F_{J}^{*}$, can be expressed in this setting as

$$
\mathcal{Q}:=\bigcup_{J \in \mathcal{H}} \sum_{i \in J} \operatorname{epi} f_{i}^{*}+\bigcup_{\lambda \in S^{+}} \operatorname{epi}(\lambda G)^{*} .
$$

The problem $\left(\mathrm{RP}_{x^{*}}\right)$ becomes here

$$
\left(\mathrm{RP}_{x^{*}}\right) \inf _{x \in X} \sup _{J \in \mathcal{H}}\left\{F_{J}\left(x, 0_{J}\right)-\left\langle x^{*}, x\right\rangle\right\},
$$


and its robust dual problem $\left(\mathrm{RD}_{x^{*}}\right)$ can be written in the following form (see (13) and (16)):

$$
\left(\mathrm{RD}_{x^{*}}\right) \quad \sup _{\substack{(J, \lambda) \in \mathcal{H} \times S^{+} \\\left(\left(x_{i}^{*}\right)_{i \in J}, u^{*}\right) \in\left(X^{*}\right)^{J} \times X^{*} \\ \sum_{i \in J} x_{i}^{*}+u^{*}=x^{*}}}-\left[\sum_{i \in J} f_{i}^{*}\left(x_{i}^{*}\right)+(\lambda G)^{*}\left(u^{*}\right)\right] .
$$

Now, for each $(J, \lambda) \in \mathcal{H} \times S^{+},\left(\left(x_{i}^{*}\right)_{i \in J}, u^{*}\right) \in\left(X^{*}\right)^{J} \times X^{*}$ such that $\sum_{i \in J} x_{i}^{*}+$ $u^{*}=x^{*}$, and each $x \in A$, we have

$$
\begin{aligned}
f(x)-\left\langle x^{*}, x\right\rangle & \geq \sum_{i \in J}\left(f_{i}(x)-\left\langle x_{i}^{*}, x\right\rangle\right)+(\lambda G)(x)-\left\langle u^{*}, x\right\rangle \\
& \geq-\left[\sum_{i \in J} f_{i}^{*}\left(x_{i}^{*}\right)+(\lambda G)^{*}\left(u^{*}\right)\right] .
\end{aligned}
$$

It follows that the weak duality relation

$$
\inf \left(\mathrm{RP}_{x^{*}}\right) \geq \sup \left(\mathrm{RD}_{x^{*}}\right)
$$

always holds (see, more generally, [8], [9]). One says that zero duality gap holds (respectively, strong duality gap holds) for the pair of primal-dual problems $\left(\mathrm{RP}_{x^{*}}\right)-$ $\left(\mathrm{RD}_{x^{*}}\right)$ if $\inf \left(\mathrm{RP}_{x^{*}}\right)=\sup \left(\mathrm{RD}_{x^{*}}\right)\left(\right.$ respectively, $\left.\inf \left(\mathrm{RP}_{x^{*}}\right)=\max \left(\mathrm{RD}_{x^{*}}\right)\right)$. These desirable duality properties are said to be stable with respect to a given set $\emptyset \neq$ $\mathcal{V} \subset X^{*}$ (also called $\mathcal{V}$-stable $)$ whenever they hold for any $x^{*} \in \mathcal{V}$.

For each $J \in \mathcal{H}$ we have, in general,

$$
\sum_{i \in J} \operatorname{epi} f_{i}^{*} \subset \operatorname{epi}\left(\square_{i \in J} f_{i}^{*}\right) \subset \operatorname{epi}\left(\sum_{i \in J} f_{i}\right)^{*} \subset \text { epi } f^{*} .
$$

On the other hand, for each $\lambda \in S^{+}$we have $\lambda G \leq \delta_{A},(\lambda G)^{*} \geq \delta_{A}^{*}$, epi $(\lambda G)^{*} \subset$ epi $\delta_{A}^{*}$, and, consequently,

$$
\bigcup_{\lambda \in S^{+}} \operatorname{epi}(\lambda G)^{*} \subset \operatorname{epi} \delta_{A}^{*}
$$

We have from (17), (20), and (21) that

$$
\mathcal{Q} \subset \text { epi } f^{*}+\operatorname{epi} \delta_{A}^{*} \subset \operatorname{epi}\left(f+\delta_{A}\right)^{*} .
$$

Proposition 1 Assume that $\left(f_{i}\right)_{i \in I} \subset \Gamma(X)$ and $\operatorname{epi}_{S} G$ is closed and convex. Then,

$$
\operatorname{epi}\left(f+\delta_{A}\right)^{*}=\overline{\operatorname{epi} f^{*}+\operatorname{epi} \delta_{A}^{*}} .
$$

Proof. One has $f \in \Gamma(X)$. Since epi ${ }_{S} G$ is convex and closed, $A \times\left\{0_{Z}\right\}=\left(\operatorname{epi}_{S} G\right) \cap$ $\left(X \times\left\{0_{Z}\right\}\right)$ is convex and closed, too. Consequently, $\delta_{A} \in \Gamma(X)$. The conclusion follows from [3, page 281].

Let us now evaluate epi $f^{*}$ and $\operatorname{epi}\left(f+\delta_{A}\right)^{*}$. 
Proposition 2 Assume that $\left(f_{i}\right)_{i \in I} \subset \Gamma(X)$. Then

$$
\text { epi } f^{*}=\overline{\mathrm{co}}\left(\bigcup_{J \in \mathcal{H}} \sum_{i \in J} \operatorname{epi} f_{i}^{*}\right) \text {. }
$$

Proof. Let $\psi:=\inf _{J \in \mathcal{H}} \square_{i \in J} f_{i}^{*}$. We then have (see [32, Theorem 2.3 .1 (ix)])

$$
\psi^{*}=\sup _{J \in \mathcal{H}} \sum_{i \in J} f_{i}^{* *}=\sup _{J \in \mathcal{H}} \sum_{i \in J} f_{i}=f
$$

and, since $\operatorname{dom} \psi^{*} \neq \emptyset, \overline{\mathrm{co}} \psi=\psi^{* *}=f^{*}$ and epi $f^{*}=\overline{\mathrm{co}}($ epi $\psi)$. By definition of $\psi$ we get

$$
\operatorname{epi}_{s} \psi \subset \bigcup_{J \in \mathcal{H}} \sum_{i \in J} \operatorname{epi} f_{i}^{*} \subset \operatorname{epi} \psi
$$

Consequently,

$$
\begin{aligned}
\overline{\mathrm{co}} \text { epi } \psi=\overline{\mathrm{co}}(\overline{\mathrm{epi} \psi}) & =\overline{\mathrm{co}}\left(\bigcup_{J \in \mathcal{H}} \sum_{i \in J} \text { epi } f_{i}^{*}\right) \\
& =\overline{\mathrm{co}}\left(\bigcup_{J \in \mathcal{H}} \sum_{i \in J} \text { epi } f_{i}^{*}\right)
\end{aligned}
$$

and the proof is complete.

Proposition 3 Assume that $\left(f_{i}\right)_{i \in I} \subset \Gamma(X)$ and $\mathrm{epi}_{S} G$ is convex closed. Then,

$$
\operatorname{epi}\left(f+\delta_{A}\right)^{*}=\overline{\operatorname{co}} \mathcal{Q} .
$$

Proof. Recall that

$$
\mathcal{Q}=\bigcup_{J \in \mathcal{H}} \sum_{i \in J} \operatorname{epi} f_{i}^{*}+\bigcup_{\lambda \in S^{+}} \operatorname{epi}(\lambda G)^{*} .
$$

In the one hand, by Property 1 , we have

$$
\operatorname{co} \mathcal{Q}=\operatorname{co}\left(\bigcup_{J \in \mathcal{H}} \sum_{i \in J} \operatorname{epi} f_{i}^{*}\right)+\bigcup_{\lambda \in S^{+}} \operatorname{epi}(\lambda G)^{*} .
$$

On the other hand, by Propositions 1 and 2, Property 2, and (23), we have

$$
\begin{aligned}
& \operatorname{epi}\left(f+\delta_{A}^{*}\right)=\overline{\operatorname{epi} f^{*}+\operatorname{epi} \delta_{A}^{*}} \\
&= \overline{\overline{\operatorname{co}}\left(\bigcup_{J \in \mathcal{H}} \sum_{i \in J} \mathrm{epi} f_{i}^{*}\right)+\overline{\bigcup_{\lambda \in S^{+}} \operatorname{epi}(\lambda G)^{*}}} \\
&=\overline{\operatorname{co}\left(\bigcup_{J \in \mathcal{H}} \sum_{i \in J} \operatorname{epi} f_{i}^{*}\right)+\bigcup_{\lambda \in S^{+}} \operatorname{epi}(\lambda G)^{*}} \\
&=\overline{\mathrm{co}} \mathcal{Q}
\end{aligned}
$$

and we are done. 
Theorem 2 (Characterization of stable strong duality) Let $\emptyset \neq \mathcal{V} \subset X^{*}$. The next statements are equivalent:

(i) $\inf \left(\mathrm{RP}_{x^{*}}\right)=\max \left(\mathrm{RD}_{x^{*}}\right), \forall x^{*} \in \mathcal{V}$.

(ii) $\operatorname{epi}\left(f+\delta_{A}\right)^{*} \cap(\mathcal{V} \times \mathbb{R})=\mathcal{Q} \cap(\mathcal{V} \times \mathbb{R})$.

If, additionally, $\left(f_{i}\right)_{i \in I} \subset \Gamma(X)$ and $\mathrm{epi}_{S} G$ is convex and closed, the following statement is equivalent to $(i)$ and $(i i)$ :

(iii) $\mathcal{Q}$ is $w^{*}$-closed convex regarding $\mathcal{V} \times \mathbb{R}$.

Proof. $[(i) \Rightarrow(i i)]$ By $(22)$, we have only to prove that inclusion [ $\subset$ ] holds in $(i i)$. Let $\left(x^{*}, s\right) \in \operatorname{epi}\left(f+\delta_{A}\right)^{*} \cap(\mathcal{V} \times \mathbb{R})$. We have $-\inf \left(\mathrm{RP}_{x^{*}}\right) \leq s$ and, by (i), there exist $(J, \lambda) \in \mathcal{H} \times S^{+}$and $\left(\left(x_{i}^{*}\right)_{i \in J}, u^{*}\right) \in\left(X^{*}\right)^{J} \times X^{*}$ such that $x^{*}=\sum_{i \in J} x_{i}^{*}+u^{*}$ and $\sum_{i \in J} f_{i}\left(x_{i}^{*}\right)+(\lambda G)^{*}\left(u^{*}\right) \leq s$.

Now there exist $\left(\left(s_{i}\right)_{i \in J}, t\right) \in \mathbb{R}^{J} \times \mathbb{R}$ such that

$$
\sum_{i \in J} s_{i}+t=s, \quad f_{i}^{*}\left(x_{i}^{*}\right) \leq s_{i} \forall i \in J, \quad(\lambda G)^{*}\left(u^{*}\right) \leq t .
$$

We then have

$$
\left(x^{*}, s\right)=\sum_{i \in J}\left(x_{i}^{*}, s_{i}\right)+\left(u^{*}, t\right) \in \sum_{i \in J} \operatorname{epi} f_{i}^{*}+\operatorname{epi}(\lambda G)^{*} .
$$

So, $\left(x^{*}, s\right) \in \mathcal{Q}$ and we are done.

$[(i i) \Rightarrow(i)]$ Take $x^{*} \in \mathcal{V}$. Assume firstly that $\inf \left(\mathrm{RP}_{x^{*}}\right)=-\infty$. By (18)-(19) we have $\sup \left(\mathrm{RD}_{x^{*}}\right)=-\infty=\max \left(\mathrm{RD}_{x^{*}}\right)$.

Since $\inf \left(\mathrm{RP}_{x^{*}}\right) \neq+\infty$, it remains the case when $r:=\inf \left(\mathrm{RP}_{x^{*}}\right) \in \mathbb{R}$. In this case, we have $\left(x^{*},-r\right) \in \operatorname{epi}\left(f+\delta_{A}\right)^{*} \cap(\mathcal{V} \times \mathbb{R})$ and, by (ii), there exist $(J, \lambda) \in \mathcal{H} \times S^{+},\left(x_{i}^{*}, s_{i}\right)_{i \in J} \in \prod_{i \in J}$ epi $f_{i}^{*}, \lambda \in S^{+}$and $\left(u^{*}, t\right) \in \operatorname{epi}(\lambda G)^{*}$ such that $\left(x^{*},-r\right)=\left(\sum_{i \in J} x_{i}^{*}+u^{*}, \sum_{i \in J} s_{i}+t\right)$. Since

$$
\begin{gathered}
-r \geq \sum_{i \in J} f_{i}^{*}\left(x_{i}^{*}\right)+(\lambda G)^{*}\left(u^{*}\right) \geq-\sup \left(\mathrm{RD}_{x^{*}}\right) \geq-r, \\
r=-\left[\sum_{i \in J} f_{i}^{*}\left(x_{i}^{*}\right)+(\lambda G)^{*}\left(u^{*}\right)\right]=\sup \left(\mathrm{RD}_{x^{*}}\right),
\end{gathered}
$$

yielding $\inf \left(\mathrm{RP}_{x^{*}}\right)=\max \left(\mathrm{RD}_{x^{*}}\right)$. As $x^{*} \in \mathcal{V}$ is taken arbitrarily, $(i)$ holds.

We now assume that $\left(f_{i}\right)_{i \in I} \subset \Gamma(X)$ and $\operatorname{epi}_{S} G$ is convex and closed.

$[(i i) \Longleftrightarrow(i i i)]$ By Proposition $3, \operatorname{epi}\left(f+\delta_{A}\right)^{*}=\overline{\operatorname{co}} \mathcal{Q}$, so that $(i i)$ is equivalent to

$$
\overline{\operatorname{co}} \mathcal{Q} \cap(\mathcal{V} \times \mathbb{R})=\mathcal{Q} \cap(\mathcal{V} \times \mathbb{R})
$$

and we are done.

Remark 3 The equivalence $[(i i) \Longleftrightarrow(i i i)]$ in Theorem 2 can also be obtained directly from Theorem 1 .

Remark 4 In the case when $\mathcal{H}$ is directed by inclusion and the functions $f_{i}, i \in I$, are non-negative, the family of convex sets $\left(\sum_{i \in J} \text { epi } f_{i}^{*}\right)_{J \in \mathcal{H}}$ is directed. Consequently, $\bigcup_{J \in \mathcal{H}} \sum_{i \in J}$ epi $f_{i}^{*}$ and $\mathcal{Q}$ are convex. In such a case, statement (iii) reads " $\mathcal{Q}$ is $w^{*}$-closed regarding $\mathcal{V} \times \mathbb{R}$ ". 
Remark 5 If $\mathcal{H}=\mathcal{F}(I)$ and $f_{i} \geq 0, i \in I, \mathcal{Q}$ is convex and $\sum_{i \in I}^{\mathcal{H}} f_{i}$ coincides with the infinite sum $\sum_{i \in I} f_{i}$ studied in various papers (see Table 1).

In the same lines as in Theorem 2 we now characterize the zero duality gap property.

Theorem 3 (Characterization of zero duality gap) Given $x^{*} \in X^{*}$, the next statements are equivalent:

(i) $\inf \left(\mathrm{RP}_{x^{*}}\right)=\sup \left(\mathrm{RD}_{x^{*}}\right)$.

(ii) $\operatorname{epi}\left(f+\delta_{A}\right)^{*} \cap\left(\left\{x^{*}\right\} \times \mathbb{R}\right)=\overline{\mathcal{Q} \cap\left(\left\{x^{*}\right\} \times \mathbb{R}\right)}$.

If, additionally, $\left(f_{i}\right)_{i \in I} \subset \Gamma(X)$ and $\operatorname{epi}_{S} G$ is convex and closed, the following statements is equivalent to $(i)$ and $(i i)$ :

(iii) $\overline{\operatorname{co}} \mathcal{Q} \cap\left(\left\{x^{*}\right\} \times \mathbb{R}\right)=\overline{\mathcal{Q} \cap\left(\left\{x^{*}\right\} \times \mathbb{R}\right)}$.

Proof. $[(i) \Rightarrow(i i)]$ Since epi $\left(f+\delta_{A}\right)^{*} \cap\left(\left\{x^{*}\right\} \times \mathbb{R}\right)$ is $w^{*}$-closed, by (22) we have only to prove that inclusion $[\subset]$ holds in $(i i)$. Let $\left(f+\delta_{A}\right)^{*}\left(x^{*}\right) \leq s$. By $(i)$, we have $-\sup \left(\mathrm{RD}_{x^{*}}\right) \leq s$ and, by definition of $\left(\mathrm{RD}_{x^{*}}\right)$, for each $n \in \mathbb{N}$ there exist $\left(J_{n}, \lambda_{n}\right) \in \mathcal{H} \times S^{+},\left(\left(x_{n, i}^{*}\right)_{i \in J_{n}}, u_{n}^{*}\right) \in\left(X^{*}\right)^{J_{n}} \times X^{*}$ such that $x^{*}=\sum_{i \in J_{n}} x_{n, i}^{*}+u_{n}^{*}$ and $\sum_{i \in J_{n}} f_{i}\left(x_{n, i}^{*}\right)+\left(\lambda_{n} G\right)^{*}\left(u_{n}^{*}\right) \leq s+\frac{1}{n}$. Now, there exist $\left(\left(s_{n . i}\right)_{i \in J_{n}}, t_{n}\right) \in \mathbb{R}^{J_{n}} \times \mathbb{R}$ such that $f_{i}\left(x_{n, i}^{*}\right) \leq s_{n . i}$, for all $i \in J_{n},\left(\lambda_{n} G\right)^{*}\left(u_{n}^{*}\right) \leq t_{n}$, and $\sum_{i \in J_{n}} s_{n . i}+t_{n}=$ $s+\frac{1}{n}$. We then have

$$
\begin{gathered}
\left(x^{*}, s+\frac{1}{n}\right) \in \sum_{i \in J_{n}} \operatorname{epi} f_{i}^{*}+\operatorname{epi}\left(\lambda_{n} G\right)^{*} \subset \mathcal{Q}, \forall n \in \mathbb{N}, \\
\left(x^{*}, s+\frac{1}{n}\right) \in \mathcal{Q} \cap\left(\left\{x^{*}\right\} \times \mathbb{R}\right), \forall n \in \mathbb{N},
\end{gathered}
$$

and, finally,

$$
\left(x^{*}, s\right) \in \overline{\mathcal{Q} \cap\left(\left\{x^{*}\right\} \times \mathbb{R}\right)} .
$$

$[(i i) \Rightarrow(i)]$ If $\inf \left(\mathrm{RP}_{x^{*}}\right)=-\infty$ then by $(18)-(19)$ we have $\sup \left(\mathrm{RD}_{x^{*}}\right)=-\infty=$ $\max \left(\mathrm{RD}_{x^{*}}\right)$. As $\inf \left(\mathrm{RP}_{x^{*}}\right) \neq+\infty$, it remains the case when $r:=\inf \left(\mathrm{RP}_{x^{*}}\right) \in \mathbb{R}$. In such a case, we have

$$
\left(x^{*},-r\right) \in \operatorname{epi}\left(f+\delta_{A}\right)^{*} \cap\left(\left\{x^{*}\right\} \times \mathbb{R}\right)
$$

and, by $(i i)$, there is a net $\left(s_{d}\right)_{d \in D}$ such that $\left(x^{*}, s_{d}\right) \in \mathcal{Q}$ for all $d \in D$ and $s_{d} \longrightarrow-r$. Now, there exist $\left(J_{d}, \lambda_{d}\right) \in \mathcal{H} \times S^{+},\left(\left(x_{d, i}^{*}\right)_{i}, s_{d, i}\right) \in$ epi $f_{i}^{*}$ for all $i \in J_{d}$ and $\left(u_{d}^{*}, t_{d}\right) \in \operatorname{epi}\left(\lambda_{d} G\right)^{*}$, such that

$$
\left(x^{*}, s_{d}\right)=\sum_{i \in J_{d}}\left(x_{d, i}^{*}, s_{d, i}\right)+\left(u_{d}^{*}, t_{d}\right)
$$

and we have

$$
s_{d}=\sum_{i \in J_{d}} s_{d, i}+t_{d} \geq \sum_{i \in J_{d}} f_{i}\left(x_{d, i}^{*}\right)+\left(\lambda_{d} G\right)^{*}\left(u_{d}^{*}\right) \geq-\sup \left(\mathrm{RD}_{x^{*}}\right) .
$$

Passing to the limit one gets $-r \geq-\sup \left(\mathrm{RD}_{x^{*}}\right)$ and we are done.

The argument for $[(i i) \Longleftrightarrow($ iii $)]$ under the additional assumption is the same as in Theorem 2 .

See [23, Corollary 1] for a deterministic counterpart of Theorem 3. 


\section{Subdifferential of the objective function of the robust problem}

We now proceed with a detailed study of the subdifferential of the objective function of $\left(\mathrm{RP}_{0_{X^{*}}}\right)$ :

$$
\left(\mathrm{RP}_{0_{X^{*}}}\right) \quad \inf _{x \in X} h(x),
$$

where $h:=f+\delta_{A}, f=\sup _{J \in \mathcal{H}} \sum_{i \in J} f_{i}$, and $A=\{x \in X: G(x) \in-S\}$.

Given $x \in \operatorname{dom} f$, consider

$$
\mathcal{H}(x):=\left\{J \in \mathcal{H}: \sum_{i \in J} f_{i}(x)=f(x)\right\} .
$$

Note that, if $\mathcal{H}=\mathcal{F}(I)$, then $\mathcal{H}(x)=\left\{J \in \mathcal{F}(I): f_{i}(x) \leq 0, \forall i \in I \backslash J\right\}$. If $\mathcal{H}=\mathcal{H}_{1}$ (the set defined in (3)), then $\mathcal{H}(x)=\left\{\{i\}: f_{i}(x)=\sup _{j \in I} f_{j}(x)\right\}$ and in this case we denote it by

$$
I(x):=\left\{i \in I: f_{i}(x)=\sup _{j \in I} f_{j}(x)\right\},
$$

which is the the so-called set of active indexes at $x$.

Proposition 4 For each $x \in \operatorname{dom} f$, we have

$$
\bigcup_{J \in \mathcal{H}(x)} \sum_{i \in J} \partial f_{i}(x) \subset \partial f(x)
$$

Proof. Let $J \in \mathcal{H}(x), x_{i}^{*} \in \partial f_{i}(x)$ for all $i \in J$, and $x^{*}:=\sum_{i \in J} x_{i}^{*}$. For any $u \in X$ we have

$$
\begin{aligned}
f(u) \geq \sum_{i \in J} f_{i}(u) & \geq \sum_{i \in J}\left(f_{i}(x)+\left\langle x_{i}^{*}, u-x\right\rangle\right) \\
& =f(x)+\left\langle x^{*}, u-x\right\rangle,
\end{aligned}
$$

which means that $x^{*} \in \partial f(x)$.

Proposition 5 For each $x \in A$ we have

$$
\bigcup_{\substack{\left.\lambda \in S^{+} \\ \lambda, G(x)\right\rangle=0}} \partial(\lambda G)(x) \subset N(A, x) .
$$

Proof. Let $\lambda \in S^{+},\langle\lambda, G(x)\rangle=0$, and $x^{*} \in \partial(\lambda G)(x)$. For any $u \in A$ we have

$$
0 \geq(\lambda G)(u) \geq(\lambda G)(x)+\left\langle x^{*}, u-x\right\rangle=\left\langle x^{*}, u-x\right\rangle,
$$

that means $x^{*} \in N(A, x)$.

Proposition 6 For each $x \in A \cap \operatorname{dom} f=\operatorname{dom} h$ we have

$$
\bigcup_{J \in \mathcal{H}(x)} \sum_{i \in J} \partial f_{i}(x)+\bigcup_{\substack{\lambda \in S^{+} \\\langle\lambda, G(x)\rangle=0}} \partial(\lambda G)(x) \subset \partial h(x) .
$$

Proof. By Propositions 4 and 5, the left hand side in (24) is contained in $\partial f(x)+$ $\partial \delta_{A}(a)$, which is contained in $\partial\left(f+\delta_{A}\right)(x)=\partial h(x)$. 
Theorem 4 (The subdifferential of the robust sum function) Let $x \in A \cap$ $\operatorname{dom} f=\operatorname{dom} h$. Next statements are equivalent:

(i) $\partial h(x)=\bigcup_{J \in \mathcal{H}(x)} \sum_{i \in J} \partial f_{i}(x)+\bigcup_{\substack{\lambda \in S^{+} \\\langle\lambda, G(x)\rangle=0}} \partial(\lambda G)(x)$.

(ii) $\left(\right.$ epi $\left.h^{*}\right) \cap(\partial h(x) \times \mathbb{R})=\mathcal{Q} \cap(\partial h(x) \times \mathbb{R})$.

If, additionally, $\left(f_{i}\right)_{i \in I} \subset \Gamma(X)$ and $\operatorname{epi}_{S} G$ is closed and convex, then the next statement is also equivalent to $(i)$ and $(i i)$ :

(iii) $\mathcal{Q}$ is $w^{*}$-closed and convex regarding $\partial h(x) \times \mathbb{R}$.

Proof. $[(i) \Rightarrow(i i)]$ By $(22)$ we have only to check the inclusion $[\subset]$ in $(i i)$. Let $x^{*} \in \partial h(x)$ and any $s \geq h^{*}\left(x^{*}\right)$. By (i), there exist $(J, \lambda) \in \mathcal{H}(x) \times S^{+},\left(x_{i}^{*}\right)_{i \in J} \in$ $\prod_{i \in J} \partial f_{i}(x)$ and $u^{*} \in \partial(\lambda G)(x)$ such that $\langle\lambda, G(x)\rangle=0, x^{*}=\sum_{i \in J} x_{i}^{*}+u^{*}$, and $\sum_{i \in J} f_{i}(x)=f(x)($ as $J \in \mathcal{H}(x))$. One has

$$
\begin{aligned}
s \geq\left\langle x^{*}, x\right\rangle-h(x) & =\left\langle\sum_{i \in J} x_{i}^{*}+u^{*}, x\right\rangle-\sum_{i \in J} f_{i}(x) \\
& =\sum_{i \in J}\left(\left\langle x_{i}^{*}, x\right\rangle-f_{i}(x)\right)+\left\langle u^{*}, x\right\rangle-(\lambda G)(x) \\
& =\sum_{i \in J} f_{i}^{*}\left(x_{i}^{*}\right)+(\lambda G)^{*}\left(u^{*}\right)
\end{aligned}
$$

(the last equality follows from (5), applied to the functions $\lambda G$ and $f_{i}$ for all $i \in J$ ). Then there exist $\left(\left(s_{i}\right)_{i \in J}, t\right) \in \mathbb{R}^{J} \times \mathbb{R}$ such that

$$
s=\sum_{i \in J} s_{i}+t, \quad f_{i}^{*}\left(x_{i}^{*}\right) \leq s_{i} \forall i \in J, \quad(\lambda G)^{*}\left(u^{*}\right) \leq t
$$

and finally,

$$
\left(x^{*}, s\right)=\sum_{i \in J}\left(x_{i}^{*}, s_{i}\right)+\left(u^{*}, t\right) \in \sum_{i \in J} \operatorname{epi} f_{i}^{*}+\operatorname{epi}(\lambda G)^{*} \subset \mathcal{Q} .
$$

$[(i i) \Rightarrow(i)]$ By Proposition 6 we have only to prove that the inclusion [ $\subset$ ] holds in (i). Let $x^{*} \in \partial h(x)$ and $s:=\left\langle x^{*}, x\right\rangle-h(x)$. We have $\left(x^{*}, s\right) \in$ epi $h^{*}$ (see (5)) and by $($ ii $)$ there exist $(J, \lambda) \in \mathcal{H} \times S^{+},\left(x_{i}^{*}, s_{i}\right)_{i \in J} \in \prod_{i \in J}$ epi $f_{i}^{*},\left(u^{*}, t\right) \in \operatorname{epi}(\lambda G)^{*}$, such that $\left(x^{*}, s\right)=\sum_{i \in J}\left(x_{i}^{*}, s_{i}\right)+\left(u^{*}, t\right)$. Now

$$
\begin{aligned}
s=\left\langle x^{*}, x\right\rangle-h(x) & \leq \sum_{i \in J}\left[\left\langle x_{i}^{*}, x\right\rangle-f_{i}(x)\right]+\left\langle u^{*}, x\right\rangle-(\lambda G)(x) \\
& \leq \sum_{i \in J} f_{i}^{*}\left(x_{i}^{*}\right)+(\lambda G)^{*}\left(u^{*}\right) \leq \sum_{i \in J} s_{i}+t=s .
\end{aligned}
$$


We then have (note that $h(x)=f(x)$ as $x \in A \cap \operatorname{dom} h$ )

$$
\begin{aligned}
0= & \left\langle x^{*}, x\right\rangle-h(x)-\sum_{i \in J} f_{i}^{*}\left(x_{i}^{*}\right)-(\lambda G)^{*}\left(u^{*}\right) \\
= & \sum_{i \in J}\left\langle x_{i}^{*}, x\right\rangle+\left\langle u^{*}, x\right\rangle-f(x)-\sum_{i \in J} f_{i}^{*}\left(x_{i}^{*}\right)-(\lambda G)^{*}\left(u^{*}\right) \\
= & \sum_{i \in J}\left[\left\langle x_{i}^{*}, x\right\rangle-f_{i}^{*}\left(x_{i}^{*}\right)-f_{i}(x)\right]+\left[\sum_{i \in J} f_{i}(x)-f(x)\right] \\
& \quad+\left[\left\langle u^{*}, x\right\rangle-(\lambda G)^{*}\left(u^{*}\right)-(\lambda G)(x)\right]+[(\lambda G)(x)] .
\end{aligned}
$$

Since all the terms in the brackets are less or equal than 0 , all these terms are equal to 0 , that means

$$
x_{i}^{*} \in \partial f_{i}(x) \forall i \in J, \quad J \in \mathcal{H}(x), \quad u^{*} \in \partial(\lambda G)(x), \quad\langle\lambda, G(x)\rangle=0,
$$

and $(i)$ holds.

$[(i i) \Longleftrightarrow(i i i)]$ It is consequence of Proposition 3 .

\section{A special case: inf-sup constrained problem}

In this section we consider the so-called inf-sup constrained problem of the following model:

$$
\left(\mathrm{MP}_{x^{*}}\right) \inf _{x \in A}\left\{\sup _{i \in I} f_{i}(x)-\left\langle x^{*}, x\right\rangle\right\}
$$

Thus, $\left(\mathrm{MP}_{0_{X^{*}}}\right)$ consists in the minimization of the sup function $\sup _{i \in I} f_{i}$ on the set $A$.

\subsection{A first perturbation approach to inf-sup constrained problem}

It is clear that $\left(\mathrm{MP}_{x^{*}}\right)$ is a special case of $\left(\mathrm{RP}_{x^{*}}\right)$ when $\mathcal{H}=\mathcal{H}_{1}$. Consequently, the dual problem $\left(\mathrm{MD}_{x^{*}}\right)$ and the qualifying set $\mathcal{Q}$ in (17) now reduce, respectively, to

$$
\left(\mathrm{MD}_{x^{*}}^{1}\right) \sup _{\substack{(i, \lambda) \in I \times S^{+} \\ u^{*} \in X^{*}}}-\left\{f_{i}^{*}\left(u^{*}\right)+(\lambda G)^{*}\left(x^{*}-u^{*}\right)\right\},
$$

and

$$
\mathcal{Q}_{1}=\bigcup_{i \in I} \operatorname{epi} f_{i}^{*}+\bigcup_{\lambda \in S^{+}} \operatorname{epi}(\lambda G)^{*} .
$$

From the results obtained in previous sections, we have

Corollary 1 (Characterization of stable strong duality) Assume that $A \cap$ $\operatorname{dom}\left(\sup _{i \in I} f_{i}\right) \neq \emptyset$ and let $\emptyset \neq \mathcal{V} \subset X^{*}$. Then the next statements are equivalent:

(i) $\inf \left(\mathrm{MP}_{x^{*}}\right)=\max \left(\mathrm{MD}_{x^{*}}^{1}\right), \forall x^{*} \in \mathcal{V}$.

(ii) epi $\left(\sup _{i \in I} f_{i}+\delta_{A}\right)^{*} \cap(\mathcal{V} \times \mathbb{R})=\mathcal{Q}_{1} \cap(\mathcal{V} \times \mathbb{R})$. 
If, additionally, $\left(f_{i}\right)_{i \in I} \subset \Gamma(X), \operatorname{epi}_{S} G$ is closed and convex, then the next statement is also equivalent to $(i)$ and $(i i)$ :

(iii) $\mathcal{Q}_{1}$ is $w^{*}$-closed and convex regarding $\mathcal{V} \times \mathbb{R}$.

Example 1 Consider the problem

$$
\begin{array}{r}
\left(\operatorname{RIP}_{x^{*}}\right) \quad \inf \left\{\sup _{i \in I} f_{i}-\left\langle x^{*}, x\right\rangle\right\} \\
\text { s.t. } g_{t}(x) \leq 0, \forall t \in T,
\end{array}
$$

with $T$ infinite and $\left(f_{i}\right)_{i \in I},\left(g_{t}\right)_{t \in T} \subset \Gamma(X)$. Let $Z$ be the lcHtvs $\mathbb{R}^{T}$ endowed with the product topology, the dual of which is the space $\mathbb{R}^{(T)}$ of the so-so-called generalized finite sequences $\lambda=\left(\lambda_{t}\right)_{t \in T}$ with only finitely many $\lambda_{t}$ different from zero. Denote by $s(\lambda)=\left\{t \in T: \lambda_{t} \neq 0\right\}$ the support of $\lambda=\left(\lambda_{t}\right)_{t \in T} \in \mathbb{R}^{(T)}$. The positive cone in $\mathbb{R}^{T}$ is $S=\left(\mathbb{R}_{+}\right)^{T}=\mathbb{R}_{+}^{T}$ and we have

$$
S^{+}=\mathbb{R}_{+}^{(T)}=\left\{\left(\lambda_{t}\right)_{t \in T} \in \mathbb{R}^{(T)}: \lambda_{t} \geq 0, \forall t \in T\right\}
$$

Set $D:=\bigcap_{t \in T} \operatorname{dom} g_{t}$ and let $G: X \longrightarrow \mathbb{R}^{T}$ be such that

$$
G(x)= \begin{cases}\left(g_{t}(x)\right)_{t \in T}, & \text { if } x \in D \\ +\infty_{Z}, & \text { else }\end{cases}
$$

whose $S$-epigraph epi $\mathbb{R}_{\mathbb{R}_{+}^{T}} G$ is convex and closed in $X \times \mathbb{R}^{T}$. Moreover, defining $\sum_{t \in s(\lambda)} \lambda_{t} g_{t}+\delta_{D}=\delta_{D}$ for $\lambda=0_{\mathbb{R}^{(T)}}$, we have,

$$
(\lambda G)(x)=\sum_{t \in s(\lambda)} \lambda_{t} g_{t}(x)+\delta_{D}(x), \forall(x, \lambda) \in X \times \mathbb{R}^{(T)} .
$$

From Corollary 1, if there exists $\bar{x} \in \operatorname{dom}\left(\sup _{i \in I} f_{i}\right) \operatorname{such}$ that $\sup _{t \in T} g_{t}(\bar{x}) \leq 0$,

$$
\inf \left(\operatorname{RIP}_{x^{*}}\right)=\max _{\substack{(i, \lambda) \in I \times \mathbb{R}_{+}^{(T)} \\ u^{*} \in X^{*}}}-\left\{f_{i}^{*}\left(u^{*}\right)+\sum_{t \in s(\lambda)}\left(\lambda_{t} g_{t}+\delta_{D}\right)^{*}\left(x^{*}-u^{*}\right)\right\}, \forall x^{*} \in X^{*},
$$

if and only if $\bigcup_{i \in I}$ epi $f_{i}^{*}+\bigcup_{\lambda \in \mathbb{R}_{+}^{(T)}} \operatorname{epi}\left(\sum_{t \in s(\lambda)}\left(\lambda_{t} g_{t}+\delta_{D}\right)^{*}\right)$ is $w^{*}$-closed and convex.

Concerning the subdifferential of the robust objective function of the reformulation of $\left(\mathrm{MP}_{0_{X^{*}}}\right)$ as an unconstrained optimization problem, $h=\sup _{i \in I} f_{i}+\delta_{A}$ we have, by Theorem 4:

Corollary 2 (Subdifferential of the constrained sup-function) Assume that $\left(f_{i}\right)_{i \in I} \subset \Gamma(X), \operatorname{epi}_{S} G$ is closed and convex and that $\mathcal{Q}_{1}$ is $w^{*}$-closed and convex. Then, for each $x \in A \cap \operatorname{dom}\left(\sup _{i \in I} f_{i}\right)$, one has

$$
\partial\left(\sup _{i \in I} f_{i}+\delta_{A}\right)(x)=\bigcup_{i \in I(x)} \partial f_{i}(x)+\bigcup_{\substack{\lambda \in S^{+} \\\langle\lambda, G(x)\rangle=0}} \partial(\lambda G)(x)
$$

where $I(x)=\left\{j \in I: f_{j}(x)=\sup _{i \in I} f_{i}(x)\right\}$. 
In the case when $G(x)=0_{Z}$ for all $x \in X$, we obtain the simplest possible formula for the subdifferential of the sup-function under a closedness assumption.

Corollary 3 (Subdifferential of the unconstrained sup-function) Assume that $\left(f_{i}\right)_{i \in I} \subset \Gamma(X)$ and $\bigcup_{i \in I}$ epi $f_{i}^{*}$ is $w^{*}$-closed and convex. Then, for each $x \in \operatorname{dom}\left(\sup _{i \in I} f_{i}\right)$ we have

$$
\partial\left(\sup _{i \in I} f_{i}\right)(x)=\bigcup_{i \in I(x)} \partial f_{i}(x)
$$

Remark 6 The set $\bigcup_{i \in I}$ epi $f_{i}^{*}$ is convex in each of the following classical cases:

1) For all $(i, j) \in I \times I$ there exists $k \in I$ such that $f_{i} \leq f_{k}$ and $f_{j} \leq f_{k}$;

2) $I$ is a convex subset of some vector space and the function $i \mapsto f_{i}(x)$ is concave for each $x \in X$.

The set $\bigcup_{i \in I}$ epi $f_{i}^{*}$ is $w^{*}$-closed if $I$ is a compact subset (of some topological space) and the function $i \mapsto f_{i}(x)$ is usc for each $x \in X$.

\subsection{A second perturbation approach to inf-sup constrained problems}

We first define another dual for the inf-sup constrained problem $\left(\mathrm{MP}_{x^{*}}\right)$ introduced in (25), whose objective function is

$$
h=f+\delta_{A}=\sup _{i \in I} f_{i}+\delta_{A}=\sup _{i \in I} f_{i}+\delta_{G^{-1}(-S)},
$$

under the following assumptions:

$$
(H) \quad\left\{\begin{array}{l}
\left(f_{i}\right)_{i \in I} \subset \Gamma(X) \\
\operatorname{epi}_{S} G \text { is convex and closed } \\
\operatorname{dom} h=A \cap \operatorname{dom} f \neq \emptyset
\end{array}\right.
$$

The uncertainty set will be the unit simplex $\Delta_{I}$ of $\mathbb{R}^{(I)}$ :

$$
\Delta_{I}:=\left\{\mu=\left(\mu_{i}\right)_{i \in I} \in \mathbb{R}^{(I)}: \mu_{i} \geq 0 \forall i \in I, \sum_{i \in s(\mu)} \mu_{i}=1\right\} .
$$

We associate with each $\mu=\left(\mu_{i}\right)_{i \in I} \in \Delta_{I}$, the lcHtvs $Y_{\mu}:=X^{s(\mu)} \times Z$ and the function $F_{\mu}: X \times X^{s(\mu)} \times Z \rightarrow \mathbb{R}_{\infty}$ defined by

$$
F_{\mu}\left(x,\left(x_{i}\right)_{i \in I}, z\right):=\sum_{i \in s(\mu)} \mu_{i} f_{i}\left(x+x_{i}\right)+\delta_{-S}(z+G(x)) .
$$

Then, for all $\left(x^{*},\left(x_{i}^{*}\right)_{i \in s(\mu)}, \mu\right) \in X^{*} \times\left(X^{*}\right)^{s(\mu)} \times \mathbb{R}^{(I)}$, one has

$$
F_{\mu}^{*}\left(x^{*},\left(x_{i}^{*}\right)_{i \in s(\mu)}, \mu\right)= \begin{cases}\sum_{i \in s(\mu)}\left(\mu_{i} f_{i}\right)^{*}\left(x_{i}^{*}\right)+(\lambda G)^{*}\left(x^{*}-\sum_{i \in s(\mu)} x_{i}^{*}\right), & \text { if } \mu \in S^{+} \\ +\infty, & \text { else. }\end{cases}
$$


So, the corresponding dual problem is (see (13))

$$
\left(\mathrm{MD}_{x^{*}}^{2}\right) \quad \sup _{\substack{(\mu, \lambda) \in \Delta_{I} \times S^{+} \\\left(\left(x_{i}^{*}\right)_{i \in s(\mu)}, u^{*}\right) \in\left(X^{*}\right) \sum^{s(\mu)} \times X^{*} \\ \sum_{i \in s(\mu)} x_{i}^{*}+u^{*}=x^{*}}}-\left(\sum_{i \in s(\mu)}\left(\mu_{i} f_{i}\right)^{*}\left(x_{i}^{*}\right)+(\lambda G)^{*}\left(u^{*}\right)\right),
$$

and the qualifying set (14) comes as

$$
\begin{aligned}
\mathcal{Q}_{2} & =\bigcup_{\mu \in \Delta_{I}} \sum_{i \in s(\mu)} \mu_{i} \operatorname{epi} f_{i}^{*}+\bigcup_{\lambda \in S^{+}} \operatorname{epi}(\lambda G)^{*} \\
& =\operatorname{co}\left(\bigcup_{i \in I} \text { epi } f_{i}^{*}\right)+\bigcup_{\lambda \in S^{+}} \operatorname{epi}(\lambda G)^{*} \\
& =\operatorname{co}\left(\bigcup_{i \in I} \text { epi } f_{i}^{*}+\bigcup_{\lambda \in S^{+}} \operatorname{epi}(\lambda G)^{*}\right)=\operatorname{co} \mathcal{Q}_{1} .
\end{aligned}
$$

So, $\mathcal{Q}_{2}$ turns out to be convex. Moreover, under $(H)$ one has, by Proposition 3,

$$
\operatorname{epi}\left(\sup _{i \in I} f_{i}+\delta_{A}\right)^{*}=\overline{\operatorname{co}} \mathcal{Q}_{1}=\overline{\mathcal{Q}}_{2},
$$

$\operatorname{dom} h \neq \emptyset$, and $F_{\mu} \in \Gamma\left(X \times X^{s(\mu)} \times Z\right)$ for all $\mu \in \Delta_{I}$. Applying Theorem 1 we then obtain (compare with Corollary 1$)$ :

Theorem 5 (Stable duality) Assume $(H)$ holds and let $\emptyset \neq \mathcal{V} \subset X^{*}$. The following statements are equivalent:

(i) $\inf \left(\mathrm{MP}_{x^{*}}\right)=\sup \left(\mathrm{MD}_{x^{*}}^{2}\right), \forall x^{*} \in \mathcal{V}$.

(ii) co $\left(\bigcup_{i \in I}\right.$ epi $\left.f_{i}^{*}\right)+\bigcup_{\lambda \in S^{+}} \operatorname{epi}(\lambda G)^{*}$ is $w^{*}$-closed regarding $\mathcal{V} \times \mathbb{R}$.

In the case when $G(x)=0_{Z}$ for all $x \in X$, we get the following corollary improving [2, Remark 12.2, p. 78].

Corollary 4 Let $\left(f_{i}\right)_{i \in I} \subset \Gamma(X)$ be a family of functions such that $\operatorname{dom}\left(\sup _{i \in I} f_{i}\right) \neq$ $\emptyset$ and let $\emptyset \neq \mathcal{V} \subset X^{*}$. Next statements are equivalent:

(i) $\begin{aligned}\left(\sup _{i \in I} f_{i}\right)^{*}\left(x^{*}\right)= & \min _{\substack{\mu \in \Delta_{I} \\\left(x_{i}^{*}\right)_{i} \in\left(X^{*}\right)^{s(\mu)}}} \sum_{i \in s(\mu)}\left(\mu_{i} f_{i}\right)^{*}\left(x_{i}^{*}\right), \forall x^{*} \in \mathcal{V} . \\ & \sum_{i \in s(\mu)} x_{i}^{*}=x^{*}\end{aligned}$

(ii) $\left.\sup _{i \in I} f_{i}\right)^{*}\left(x^{*}\right)=\min _{\substack{\mu \in \Delta_{I} \\\left(x_{i}^{*}\right)_{i} \in\left(X^{*} s(\mu)\right.}} \sum_{i \in s(\mu)} \mu_{i} f_{i}^{*}\left(\frac{x_{i}^{*}}{\mu_{i}}\right), \forall x^{*} \in \mathcal{V}$.

(iii) $\operatorname{co}\left(\bigcup_{i \in I}\right.$ epi $\left.f_{i}^{*}\right)$ is $w^{*}$-closed regarding $\mathcal{V} \times \mathbb{R}$.

Let us now go back to the subdifferential of the objective function

$$
h=\sup _{i \in I} f_{i}+\delta_{A}
$$

with $A=G^{-1}(-S)$. 
Theorem 6 (Subdifferential of the sup-function) Assume $(H)$ holds and let $x \in$ $A \cap \operatorname{dom} f=\operatorname{dom} h$. Denote $I(x)=\left\{i \in I: f_{i}(x)=f(x)\right\}$. Then the next statements are equivalent:

(i) $\partial h(x)=\operatorname{co}\left(\bigcup_{i \in I(x)} \partial f_{i}(x)\right)+\bigcup_{\substack{\lambda \in S^{+} \\\langle\lambda, G(x)\rangle=0}} \partial(\lambda G)(x)$.

(ii) co $\left(\bigcup_{i \in I}\right.$ epi $\left.f_{i}^{*}\right)+\bigcup_{\lambda \in S^{+}} \operatorname{epi}(\lambda G)^{*}$ is $w^{*}$-closed regarding $\partial h(x) \times \mathbb{R}$.

Proof. $[(i) \Rightarrow(i i)]$ Let $\left(x^{*}, s\right) \in \overline{\mathcal{Q}}_{2} \cap(\partial h(x) \times \mathbb{R})$. We have to prove that $\left(x^{*}, s\right) \in \mathcal{Q}_{2}$. As $x^{*} \in \partial h(x)$, by (i) there exist $\theta=\left(\theta_{i}\right)_{i} \in \mathbb{R}_{+}^{(I(x))},\left(\left(x_{i}^{*}\right)_{i}, u^{*}\right) \in\left(X^{*}\right)^{s(\theta)} \times X^{*}$, and $\lambda \in S^{+}$such that $\sum_{i \in s(\theta)} \theta_{i}=1, x_{i}^{*} \in \partial f_{i}(x)$ for all $i \in s(\theta), u^{*} \in \partial(\lambda G)(x)$, $\langle\lambda, G(x)\rangle=0$, and $x^{*}=\sum_{i \in s(\theta)} \theta_{i} x_{i}^{*}+u^{*}$. On the other hand, as $(H)$ holds, according to (27), we have $\overline{\mathcal{Q}}_{2}=$ epi $h^{*}$, and hence, $\left(x^{*}, s\right) \in \overline{\mathcal{Q}}_{2}$ also means that $s \geq h^{*}\left(x^{*}\right) \geq\left\langle x^{*}, x\right\rangle-h(x)=\left\langle x^{*}, x\right\rangle-f(x)$. After all, as $(\lambda G)(x)=0$, we have

$$
\begin{aligned}
s \geq h^{*}\left(x^{*}\right) & \geq\left\langle x^{*}, x\right\rangle-h(x)=\left\langle x^{*}, x\right\rangle-f(x) \\
& =\sum_{i \in s(\theta)} \theta_{i}\left(\left\langle x_{i}^{*}, x\right\rangle-f_{i}(x)\right)+\left\langle u^{*}, x\right\rangle-(\lambda G)(x) \quad(\text { as } i \in I(x)) \\
& =\sum_{i \in s(\theta)} \theta_{i} f_{i}^{*}\left(x_{i}^{*}\right)+(\lambda G)^{*}\left(u^{*}\right) \quad(\text { see }(5)) .
\end{aligned}
$$

So, there exists $\left(\left(s_{i}\right)_{i}, t\right) \in \mathbb{R}^{s(\theta)} \times \mathbb{R}$ such that

$$
s=\sum_{i \in s(\theta)} s_{i}+t, \quad s_{i} \geq \theta_{i} f_{i}^{*}\left(x_{i}^{*}\right), \quad \forall i \in s(\theta), \quad \text { and } t \geq(\lambda G)^{*}\left(u^{*}\right),
$$

and we then have

$$
\left(x^{*}, s\right)=\sum_{i \in s(\theta)} \theta_{i}\left(x_{i}^{*}, \frac{s_{i}}{\theta_{i}}\right)+\left(u^{*}, t\right)
$$

yielding

$$
\left(x^{*}, s\right) \in \operatorname{co}\left(\bigcup_{i \in I(x)} \operatorname{epi} f_{i}^{*}\right)+\operatorname{epi}(\lambda G)^{*} \subset \mathcal{Q}_{2} .
$$

$[(i i) \Rightarrow(i)]$ It follows from Proposition 6 (applied to the case $\mathcal{H}=\mathcal{H}_{1}$ ) that

$$
\bigcup_{i \in I(x)} \partial f_{i}(x)+\bigcup_{\substack{\lambda \in S^{+} \\\langle\lambda, G(x)\rangle=0}} \partial(\lambda G)(x) \subset \partial h(x) .
$$

Since $\partial h(x)$ is convex it follows that the inclusion [D] always holds in $(i)$. Let us prove the reverse inclusion. Let $x^{*} \in \partial h(x)$ and $s:=\left\langle x^{*}, x\right\rangle-h(x)$. Then $\left(x^{*}, s\right) \in$ epi $h^{*}($ see $(5))$ and by $(27),\left(x^{*}, s\right) \in \overline{\mathcal{Q}}_{2}$. So, $\left(x^{*}, s\right) \in \overline{\mathcal{Q}}_{2} \cap(\partial h(x) \times \mathbb{R})$, and by $(i i)\left(x^{*}, s\right) \in \mathcal{Q}_{2}$. Then there exist $\mu=\left(\mu_{i}\right)_{i \in s(\mu)} \in \Delta_{I},\left(\left(x_{i}^{*}, s_{i}\right)\right)_{i} \in \prod_{i \in s(\mu)} \operatorname{epi} f_{i}^{*}$, 
$\lambda \in S^{+}$, and $\left(u^{*}, t\right) \in \operatorname{epi}(\lambda G)^{*}$ such that $\left(x^{*}, s\right)=\sum_{i \in s(\mu)} \mu_{i}\left(x_{i}^{*}, s_{i}\right)+\left(u^{*}, t\right)$. We have

$$
\begin{aligned}
s & =\sum_{i \in s(\mu)} \mu_{i} s_{i}+t \\
& \geq \sum_{i \in s(\mu)} \mu_{i} f_{i}^{*}\left(x_{i}^{*}\right)+(\lambda G)^{*}\left(u^{*}\right) \\
& \geq \sum_{i \in s(\mu)} \mu_{i}\left(\left\langle x_{i}^{*}, x\right\rangle-f_{i}(x)\right)+\left\langle u^{*}, x\right\rangle-(\lambda G)(x) \\
& =\left\langle x^{*}, x\right\rangle-\sum_{i \in s(\mu)} \mu_{i} f_{i}(x)-(\lambda G)(x) \\
& \geq\left\langle x^{*}, x\right\rangle-f(x)=\left\langle x^{*}, x\right\rangle-h(x)=s .
\end{aligned}
$$

Consequently,

$$
\begin{aligned}
0= & \left\langle x^{*}, x\right\rangle-f(x)-\sum_{i \in s(\mu)} \mu_{i} f_{i}^{*}\left(x_{i}^{*}\right)-(\lambda G)^{*}\left(u^{*}\right) \\
= & \sum_{i \in s(\mu)}\left[\mu_{i}\left(\left\langle x_{i}^{*}, x\right\rangle-f_{i}(x)-f_{i}^{*}\left(x_{i}^{*}\right)\right)\right]+\sum_{i \in s(\mu)}\left[\mu_{i}\left(f_{i}(x)-f(x)\right)\right] \\
& \quad+\left[\left\langle u^{*}, x\right\rangle-(\lambda G)(x)-(\lambda G)^{*}\left(u^{*}\right)\right]+[(\lambda G)(x)]
\end{aligned}
$$

We note that each term in the brackets is less or equal to 0. Since the sum of these terms is equal to 0 each of these terms is equal to 0 , that means respectively:

$$
x_{i}^{*} \in \partial f_{i}(x), \quad \forall i \in s(\mu), \quad s(\mu) \subset I(x), \quad u^{*} \in \partial(\lambda G)(x), \text { and } \quad\langle\lambda, G(x)\rangle=0 .
$$

We then have:

$$
\begin{aligned}
x^{*}=\sum_{i \in s(\mu)} \mu_{i} x_{i}^{*}+u^{*} & \in \operatorname{co}\left(\bigcup_{i \in s(\mu)} \partial f_{i}(x)\right)+\partial(\lambda G)(x) \\
& \subset \operatorname{co}\left(\bigcup_{i \in I(x)} \partial f_{i}(x)\right)+\partial(\lambda G)(x)
\end{aligned}
$$

and we are done.

Taking $G(x)=0_{Z}$ for all $x \in X$, we obtain:

Corollary 5 Assume that $\left(f_{i}\right)_{i \in I} \subset \Gamma(X)$ and

$$
\operatorname{co}\left(\bigcup_{i \in I} \text { epi } f_{i}^{*}\right) \text { is } w^{*} \text {-closed in } X^{*} \times \mathbb{R} \text {. }
$$

Then, for each $x \in \operatorname{dom}\left(\sup _{i \in I} f_{i}\right)$ we have

$$
\partial\left(\sup _{i \in I} f_{i}\right)(x)=\operatorname{co}\left(\bigcup_{i \in I(x)} \partial f_{i}(x)\right) .
$$


Remark 7 Formula (29) is to be compared with the one of Corollary 3 and also with the classical Valadier formula ([28, Theorem 2], [15, Theorem 3, p.201], [32, Theorem 2.4.18]) in which, under some continuity assumption, the $w^{*}$-closed convex hull appears in the right hand side of (29) (see however, [15, Theorem 4, p.204] and [31, Theorem 2.4]).

Remark 8 The formulas given in Corollary 3 and Corollary 5 remain valid if one replaces the assumption $\left(f_{i}\right)_{i \in I} \subset \Gamma(X)$ by the weaker one $\left(\sup _{i \in I} f_{i}\right)^{* *}=\sup _{i \in I} f_{i}^{* *}$. This last condition has been considered in [21] and [22].

To conclude, let us give two typical examples when Condition (28) is satisfied and, consequently, (29) holds. To this end, recall that the recession function $\varphi_{\infty}$ of $\varphi \in \Gamma\left(\mathbb{R}^{n}\right)$ defined by epi $\left(\varphi_{\infty}\right)=(\text { epi } \varphi)_{\infty}$, coincides with the support function of $\operatorname{dom} \varphi^{*}\left(\right.$ or $\left.\overline{\operatorname{dom} \varphi^{*}}\right)$, see [26, Theorem 13.3]. So, $h_{\infty}=\left(\delta_{\operatorname{dom} \varphi^{*}}\right)^{*}=\left(\delta \overline{\operatorname{dom} \varphi^{*}}\right)^{*}$. In both examples below, we assume that $\left(f_{i}\right)_{i \in I} \subset \Gamma\left(\mathbb{R}^{n}\right), I$ is compact, and, for each $x \in \mathbb{R}^{n}$, the function $i \mapsto f_{i}(x)$ is usc.

Example 2 Assume that the epigraphs of the conjugate functions $f_{i}^{*}, i \in I$, have the same recession cone, that means

$$
D:=\overline{\operatorname{dom} f_{i}}=\overline{\operatorname{dom} f_{j}}, \quad \forall(i, j) \in I \times I,
$$

and assume that epi $\delta_{D}^{*}$ does not contain lines. Then by [25, Theorem 5.3(b)], we have

$$
\begin{aligned}
\operatorname{epi}\left(\sup _{i \in I} f_{i}\right)^{*} & =\operatorname{co}\left(\bigcup_{i \in I} \operatorname{epi} f_{i}^{*}\right)+\operatorname{co}\left(\bigcup_{i \in I}\left(\operatorname{epi} f_{i}^{*}\right)_{\infty}\right) \\
& =\operatorname{co}\left(\bigcup_{i \in I} \operatorname{epi} f_{i}^{*}\right)+\operatorname{epi} \delta_{D}^{*}=\operatorname{co}\left(\bigcup_{i \in I} \operatorname{epi} f_{i}^{*}+\operatorname{epi} \delta_{D}^{*}\right) \\
& =\operatorname{co} \bigcup_{i \in I}\left(\operatorname{epi} f_{i}^{*}+\operatorname{epi} \delta_{D}^{*}\right)=\operatorname{co} \bigcup_{i \in I}\left[\operatorname{epi} f_{i}^{*}+\left(\operatorname{epi} f_{i}^{*}\right)_{\infty}\right] \\
& =\operatorname{co}\left(\bigcup_{i \in I} \operatorname{epi} f_{i}^{*}\right)
\end{aligned}
$$

which is closed since epi $\left(\sup _{i \in I} f_{i}\right)^{*}$ is closed.

In the case when the convex functions $f_{i}, i \in I$, are real-valued on $\mathbb{R}^{n}$ we have $\left(\text { epi } f_{i}^{*}\right)_{\infty}=\left\{0_{\mathbb{R}^{n}}\right\} \times \mathbb{R}_{+}$, for all $i \in I$, and epi $\delta_{D}^{*}$ does not contain lines. Consequently, (29) holds (see [14, Theorem 4.4.2] where the additional but nonnecessary condition $\operatorname{dom}\left(\sup _{i \in I} f_{i}\right)=\mathbb{R}^{n}$ is required).

Example 3 By [27, Corollary 5.3] we know that (28) holds if (30) holds and for each $(x, r) \in \mathbb{R}^{n} \times \mathbb{R}$ the function $i \mapsto\left(\delta_{\text {epi } f_{i}^{*}}\right)^{*}(x, r)$ is usc. We have (see [4, p.3])

Assuming that

$$
\left(\delta_{\text {epi } f_{i}^{*}}\right)^{*}(x, r)= \begin{cases}+\infty & \text { if } r>0, \\ \left(\delta_{\operatorname{dom} f_{i}^{*}}\right)^{*}(x) & \text { if } r=0, \\ -r f_{i}\left(-\frac{x}{r}\right) & \text { if } r<0 .\end{cases}
$$

$$
\overline{\operatorname{dom} f_{i}^{*}}=\overline{\operatorname{dom} f_{j}^{*}}, \quad \forall(i, j) \in I \times I,
$$

we obtain that $i \mapsto\left(\delta_{\text {epi } f_{i}^{*}}\right)^{*}(x, r)$ is usc. Consequently, if (30) and (31) hold then (29) holds. 


\section{References}

1. Aragón-Artacho, F.J., Goberna, M.A., López, M.A., Rodríguez, M.M.L.: Nonlinear Optimization. Springer, Cham (2019)

2. Bot, R.I.: Conjugate Duality in Convex Optimization. Springer, Berlin (2010)

3. Burachik, R., Jeyakumar, V.: A dual condition for the convex subdifferential sum formula with applications. J. Convex Anal. 12, 279-290 (2005)

4. Castaing, C., Valadier, M.: Convex Analysis and Measurable Multifunctions. Springer, Berlin (1997)

5. Correa, R., Hantoute, A., López, M.A.: Valadier-like formulas for the supremum function I. J. Convex Anal. 25, 1253-1278 (2018)

6. Correa, R., Hantoute, A., López, M.A.: Moreau-Rockafellar type formulas for the subdifferential of the supremum function. SIAM J. Optim. 29, 1106-1130 (2019)

7. Correa, R., Hantoute, A., López, M.A.: Valadier-like formulas for the supremum function II: the compactly indexed case. J. Convex Anal. 26, 299-324 (2019)

8. Dinh, N., Goberna, M.A., López, M.A., Volle, M.: Convexity and closedness in stable robust duality. Optim. Letters 13, 325-339 (2019)

9. Dinh, N., Goberna, M.A., López, M.A., Volle, M.: Characterizations of robust and stable duality for linear perturbed uncertain optimization problems. In: Burachik, R., Li, G. (Eds.), Proceedings of the Jonathan Borwein Commemorative Conference, 25-29 September 2017, to appear.

10. Dinh, N., Goberna, M.A., Volle, M.: Duality for the robust sum of functions. Set Val. Var Anal, to appear. DOI: 10.1007/s11228-019-00515-2

11. Dinh, N., Goberna, M.A., Volle, M.: Primal-dual optimization conditions for the robust sum of functions with applications. Appl. Math. Optim., to appear. DOI: 10.1007/s00245019-09596-9

12. Dinh, N., Mo, T.H., Vallet, G. Volle, M.: A unified approach to robust Farkas-type results with applications to robust optimization problems. SIAM J. Optim 20, 2540- 2559 (2010)

13. Goberna, M.A., Hiriart-Urruty, J.-B., López, M.A.: Best approximate solutions of inconsistent linear inequality systems. Vietnam J. Math. 46, 271-284 (2018)

14. Hiriart-Urruty, J.-B.: Lemarechal, Convex Analysis and Minimization Algorithms I: Fundamentals. Springer, Berlin, 1996.

15. Ioffe, A.D., Tihomirov, V.M.: Theory of Extremal Problems, North-Holland, AmsterdamNew York (1979)

16. Ioffe, A.D., Levin, V.L.: Subdifferentials of convex functions (Russian). Trudy Moskov Math. Obshch 26, 3-73 (1972)

17. Jeyakumar, V., Wu, Z.Y., Lee, G.M., Dinh, N.: Liberating the subgradient optimality conditions from constraint qualifications. J. Global Optim. 36, 127-137 (2006)

18. Li, G.Y., Jeyakumar, V., Lee, G.M.: Robust conjugate duality for convex optimization under uncertainty with application to data classification. Nonlinear Anal. 74, 2317-2341 (2011)

19. Li, G.Y., Ng, K.F.: On extension of Fenchel duality and its application. SIAM J. Optim. 19, 1498-1509 (2008)

20. Le Roux, S., Pauly, A.: Infinite sequential games with real-valued payoffs. Proc. of the Joint Meeting of the 23rd EACSL Annual Conference on Computer Science Logic (CSL) and the 29th Annual ACM/IEEE Symposium on Logic in Computer Science (LICS), Article No. 62, 10 pp., ACM, New York (2014)

21. López, M.A., Volle, M.: A formula for the set optimal solutions of a relaxed minimization problem. Application to subdifferential calculus. J. Conv. Anal. 17, 1057-1075 (2010)

22. López, M.A., Volle, M.: On the subdifferential of the supremum of an arbitrary family of extend real-valued functions. RACSAM 105, 3-21 (2011)

23. Luc, D.T., Volle, M.: On epsilon-stability in optimization. Vietnam J. Math. 46, 149-167 (2018)

24. Luc, D.T., Volle, M.: Duality for optimization problems with infinite sums. SIAM J. Optim. 29, 1819-1843 (2019)

25. Pérez-Aros, P.: Formulae for the conjugate and the subdifferential of the supremum function. J. Optim. Theory Appl. 180, 397-427 (2019)

26. Rockafellar, Convex Analysis, Princeton University Press, Princeton, New Jersey (1970)

27. Solov'er, V.N.: The subdifferential and the directional derivatives of the maximum of a family of convex functions. Izvestiga RAN: Ser. Mat. 62, 807-832 (1998) 
28. Valadier, M.: Sous-différentiels d'une borne supérieure et d'une somme continue de fonctions convexes (French). C. R. Acad. Sci. Paris, Sér. A-B Math. 268, 39-42 (1969)

29. Vallée, C., Zalinescu, C.: Series of convex functions: Subdifferential, conjugate and applications to entropy minimization. J. Convex Anal. 23, 1137-1160 (2016)

30. Van Ryzin, J.: Repetitive play in finite statistical games with unknown distributions. Ann. Math. Statist. 37 (1966), 976-994.

31. Volle, M.: Calculus rules for global approximate minima and applications to approximate subdifferential calculus. J. Global Optim. 5, 131-157 (1994)

32. Zalinescu, C.: Convex Analysis in General Vector Spaces, World Scientific, Singapore (2002)

33. Zheng, X.Y.: A series of convex functions on a Banach space. Acta Mathematica Sinica 4, 19-28 (1998)

34. Zheng, X.Y., Ng. K.F.: Error bound moduli for conic convex systems on Banach spaces. Math. Oper. Res. 29, 213-228 (2004) 